ES15

\title{
Lessons learned from the thermal design of an instrument (EIT, the Extreme-UV Imaging Telescope) on board SOHO
}

\author{
J.M. Defise', P. Rochus ${ }^{2}$ \\ Centre Spatial de Liège, University of Liège
}

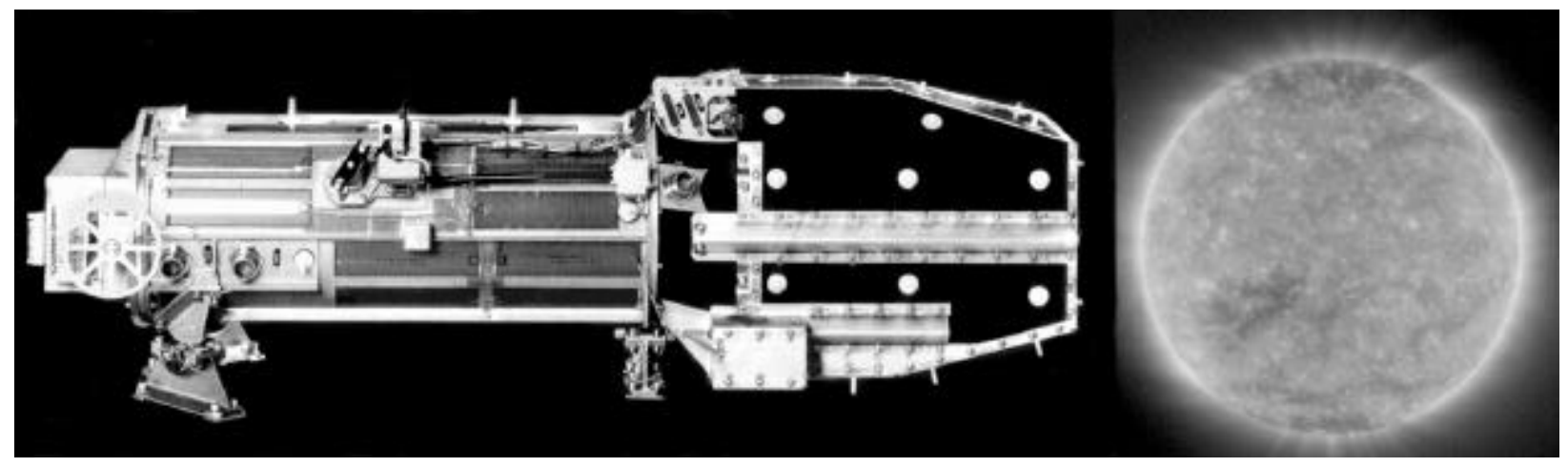

\section{ABSTRACT}

A global optical survey of the solar corona (ref 1 ) is presently accomplished by observations made with an Extreme ultraviolet Imaging Telescope (EIT), an experiment on-board of SOHO. The thermal issue was a main concern for this sensor. The thermal behaviors of the instrument and some subsystems have been extensively analyzed and tested; these can now be compared with the real behavior in space. These analyses and tests are reported. We outline the lessons learned from this experience for the thermal design of future scientific instruments and suggest improvements in the presently used methods as well as hand calculation methods which enable to easily model some specific problems and more directly extract the physical aspects of the problem.

\section{INTRODUCTION}

A global optical survey of the solar corona is presently accomplished by observations made with an Extreme ultraviolet Imaging Telescope (EIT), an experiment on-board of SOHO. EIT is a high resolution, wide field, multi-bandpass, Ritchey-Chretien telescope providing in the focal plane, images of the solar disk at four wavelengths in the EUV range corresponding to different temperatures of the corona ( He II-30.4 nm-6 $10^{4} \mathrm{~K} ; \mathrm{Fe}$ IX-17.1 nm-106 $\mathrm{K}$; Fe XII-19.5 nm-1.6 $10^{6}$ $\mathrm{K}$; Fe XV-28.4 nm-3 $10^{6} \mathrm{~K}$ ). The images in four narrow bandpasses are obtained using 4 normal incidence multilayered coatings, deposited on quadrants of the mirrors. They are recorded on a specific CCD camera especially developed for EIT. The main thermal challenges were (ref 2):

\footnotetext{
${ }^{1}$ Project engineer, Centre Spatial de Liège (Belgium)

${ }^{2}$ Director of $R \& D$ department, Centre Spatial de Liège (Belgium)
} 
- the thermal control of the optical structure,

Special care was taken to ensure the correct spacing between the telescope mirrors to maintain the optical quality. The two mirrors are mounted on each end of an aluminium cylinder, itself held to the main structure by a unique central flange to avoid thermal stresses. The on-ground optical alignment is optimized for a $20^{\circ} \mathrm{C}$ environmental temperature. Once in orbit, due to the remaining inaccuracies of the thermal design, aging effects on the thermal properties and sun flux variations, the equilibrium internal temperature will not be $20^{\circ} \mathrm{C}$ during all the observation periods. Thus, the optical cylinder has been wrapped with thermofoil heaters and a set of thermistors. The thermal design has been computed and tested to reach a temperature lower than $20^{\circ} \mathrm{C}$ in all the in-orbit situations without heater. The installed operational power on the optical cylinder shall be sufficient to increase the temperature to $20^{\circ} \mathrm{C}$ with the 5 Watts max allocated resources. Thus, the distance between the primary and the secondary mirror is made constant by means of this active thermal control. This configuration is the result of a trade-off study with the use of an Invar internal tube which has two drawbacks: more important mass and possible expansion due to aging effects independent on temperature changes.

\section{Aluminum filter, \\ - the thermal behavior of the entrance}

At the level of the entrance of the instrument, a set of wide aluminum filters is mounted in order to block solar visible and IR light, and to settle a well controlled internal thermal environment. To provide a better mechanical strength, they are made with a 60 $\mathrm{nm}$ plastic film and a wide supporting $\mathrm{Ni}$ grid $(5 \times 5$ $\mathrm{mm}$ grid, $100 \mu \mathrm{m}$ wide) encased between two 150 $\mathrm{nm}$ thick aluminum foils. They ensure a rejection of the visible better than $10^{5}$. Moreover, the carbon contained in the plastic is used to reject light at wavelengths longer than $500 \AA$. A transmission close to $40 \%$ is achieved in the band passes of the instrument. The lifetime of this filter is strongly dependent on the temperature reached in front of the sun.

\section{- the cooling of the detector down to $-80^{\circ} \mathrm{C}$.}

The CCD detector requires a very low operational temperature, around $-80^{\circ} \mathrm{C}$. This is to reduce the dark current to an acceptable value and mainly to increase the CTE by reducing the influence of the lattice defects induced by radiations. To achieve this requirement, the CCD is conductively connected to an external radiator with an important view factor with space. The selection of the radiator coating has been done to provide the highest heat rejection, it is a specific yellow paint with a high emissivity. To improve the efficiency of the cooling, an additional shield mainly specularly reflective is mounted close to the radiator, directly on the platform of the $\mathrm{S} / \mathrm{C}$, to hide the hottest spacecraft parts still in the field of view.

The thermal behaviors of the instrument and of some subsystems have been extensively analyzed and tested; these can now be compared with the real behavior in space. These analyses, tests and correlations are shortly reported. We outline the lessons learned from this experience for the thermal design of future scientific instruments. It is for example mandatory to evaluate at the earliest stage of the project, the real thermal behavior of critical areas such as contact conductances, MLI efficiency, shading effects, multiple reflections in photon traps appearing in baffles for example... This will avoid late additional thermal tests. Specific tests should be foreseen in the schedule to experimentally evaluate these parameters.

Improvements in the presently used methods as well as hand calculation methods are proposed. These suggested methods are based on the following theoretical developments:

- Thevenin - Norton theorem in its general form is used to generalize the usual way to specify the thermal environment of a specific instrument on a spacecraft, with two numbers: a sink temperature and an effective emissivity for each external surface. This more complete definition of the thermal environment of an instrument is more judicious for external surfaces of the same instrument, which are strongly radiatively coupled. A method to generate an equivalent reduced thermal mathematical model from the complete thermal mathematical model is suggested as well as a method to define the thermal balance test environment for a given geometry of thermal shrouds around the experiment.

- The Gebhart factor theory is extended for a system exclusively composed of partly diffuse/partly specular, gray surfaces with the aim to deliver a method which allows hand calculations, enables to easily modelize some specific problems we encountered like:

+ simple modelisation of the multiple

reflections in photon traps appearing in baffle which leaded to a high temperature for the door and for the filter. (A similar problem appeared on SOHO where the temperature of the Fine Pointing Sun Sensor was hotter than foreseen due to multiple reflections in a "cavity" created by the experiments around it). To avoid this type of problems, the gaps between instruments and S/C must be closed by MLI skirts in order to avoid sun trapping and unpredictable multiple refelctions within the gaps. 
+ a better cooling efficiency of the radiator, with an additional specular shield, mounted close to it, directly on the platform of the $S / C$, to hide the hottest parts of the spacecraft still in the field of view.

Hand calculation methods (ref 3 ) can generally be used for initial design analysis, comprehensive model checking, and emphasize the main physical contributions of a given problem. It has now been recognized that radiant heat transfer between surfaces of a specular character is perhaps the more common situation.

\section{THE EIT INSTRUMENT}

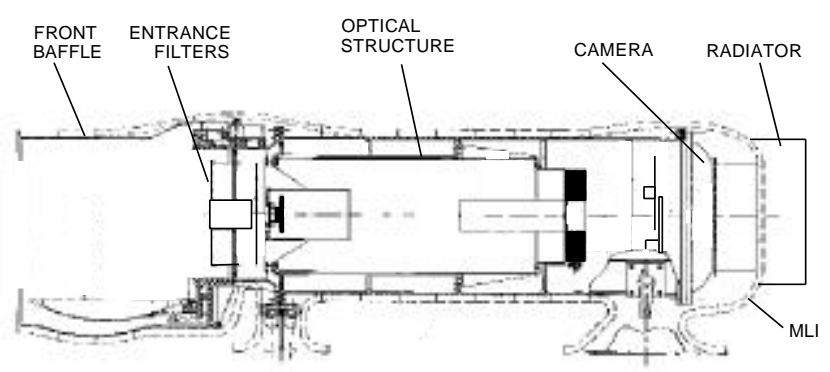

Figure (1.): Layout of the EIT instrument

Among the 11 solar experiments mounted on the SOHO platform, EIT is a medium imaging telescope of the $15 \mathrm{~kg}$ class. The EIT experiment is a multibandpass telescope (ref 4) providing in the focal plane images of the solar disk at four wavelengths in the EUV range ( He II-30.4 nm-6 $10^{4} \mathrm{~K}$; Fe IX-17.1 nm-106 K ; Fe XII-19.5 nm-1.6 $10^{6} \mathrm{~K}$; Fe XV-28.4 nm-3 $10^{6} \mathrm{~K}$ ). The experiment is a high resolution, wide field telescope that will produce images of the corona on the disk and above the solar limb.

The telescope is a Ritchey-Chretien system, with 2 superpolished mirrors accurately aligned on an optical metering tube. The mirrors are made of Zerodur, and have an interferential multilayered coating used to select 4 narrow bandpasses in the EUV range.

Launched on December 95 the $2^{\text {nd }}$, the $\mathrm{SOHO}$ spacecraft is orbiting around the L1 libration point, at $1,500,000 \mathrm{~km}$ from the earth, on the earth-sun line. The 3 -axes stabilized spacecraft is permanently pointing the sun within 10 arcsec with a short term stability of 1 arsec in 15 minutes, providing a very stable thermal environment without any terrestrial or lunar eclipse. The sun flux is continuously oriented toward the entrance of the EIT instrument. The solar flux is then the driving parameter of the very specific thermal environment of SOHO.

\section{THERMAL CONTROL OF THE OPTICAL STRUCTURE}

\section{SCOPE}

The optical structure is made of the optical cylinder, the 2 mirrors and their respective baffle.

The primary mirror (diameter $120 \mathrm{~mm}$ ) is held by 3 preloaded Invar flexible blades and 3 spherical bearings. This type of mounting is used to reduce to its minimum the thermo-mechanical stresses in the Zerodur and to ensure a stable mounting. Its main drawback appeared during vibration qualification, where the primary mirror showed high vibration resonances in the high frequency range.

The secondary mirror is smaller. Its tail is glued in an Invar support with specific metrological controls, Invar having the same expansion coefficient as Zerodur. The secondary support is screwed on a aluminum spider. This spider is then shimmed and screwed on the optical cylinder once the alignment is reached.

The optical cylinder has to comply with the following requirements:

\section{good mechanical resistance}

avoid any non-symmetrical thermal deformations (no thermal tilt on the mirrors)

shall not produce any stress on the

mirrors

shall be compliant with the vibration behavior of the instrument and the qualification limits on the mirrors

Thus, the mounting interfaces of the cylinder has been limited to a central flange, located at the center of mass of the complete system. This provides a quasi-isostatic holding of the cylinder, reducing the thermal conductive path to the external structure and limiting it to one conductive boundary condition. But again, as a drawback, from the vibration point of view, this solution is not the optimum to damp out the input vibrations of the main structure.

The on-ground alignment was a very critical process that finally achieved a correct tilting and a very accurate on-axis spacing of both mirrors. Interferometric methods have been used for this purpose. For stability and mechanical purposes, the 2 mirrors are screwed and maintained in their aligned position, after the onground alignment. The on-ground alignment was performed in a standard clean room, in a $20^{\circ} \mathrm{C}$ 
environment. This defines specific requirements for the thermal design.

There is no mechanical adjustment for further in-orbit realignment. It means that the thermomechanical behavior of the optical system must be compliant with all the alignment requirements, and that a continuous $20^{\circ} \mathrm{C}$ operational temperature must be achieved on the optical system.

\section{THE OPTICAL SYSTEM THERMAL REQUIREMENTS:}

The thermal requirements of the optical structure can be listed as follow:

The primary-secondary distance along the optical axis: with the aluminum cylinder, temperature excursions around $20^{\circ} \mathrm{C}$ shall be restricted to half a degree, i. e. thermal range is $\left[19.5^{\circ} \mathrm{C}, 20.5^{\circ} \mathrm{C}\right]$ during all the operational life of the instrument.

The mounting of the secondary mirror, with Invar shall not produce thermal stresses at the Invar/aluminum interface, i.e. non-operational temperature range shall remain close to $20^{\circ} \mathrm{C}$.

- Keep a good uniformity of the temperature to ensure an adequate thermo-elastic stability.

A lot of parameters are to be taken into account for the thermal design:

- $\quad$ aging of sun-exposed surfaces

- $\quad$ solar flux variations; the SOHO mission is scheduled to start beginning of 1996 (solar minimum, $1350 \mathrm{~W} / \mathrm{m}^{2}$ ) and will see an increase until 2001 (solar maximum, $1450 \mathrm{~W} / \mathrm{m}^{2}$ )

- temperature variations of the $\mathrm{SOHO}$ platform, thermally controlled to ensure a pointing control $\left[+10^{\circ} \mathrm{C},+30^{\circ} \mathrm{C}\right]$

temperature variations of the other external surfaces of SOHO (variations of the heat sink temperatures)

variation of the power dissipation in the
camera electronics due to aging of electronic
components

- $\quad$ the allocated power for a heater system is limited to $5 \mathrm{~W}$

- margins imposed by ESA on all the external SOHO heat sink temperatures $\left( \pm 8^{\circ} \mathrm{C}\right)$.

limited conductive heat flux through the attachment legs. To obtain this thermal conductivity of each foot, we measured the electrical resistance of each foot and we made use of Franz-Wiedemann relation for metals:

electrical conductivity

$$
\frac{k_{\text {therm }, e^{-}}}{\sigma_{\text {electr, } e^{-}}}=L \cdot T \text { which relates thermal to }
$$

All these effects combined to each other, lead us to foresee two extremes: a cold, beginning of life (BOL) environment for the EIT, and a warm, end of life (EOL) environment.

The final design includes an active thermal control of the optical cylinder. Thermofoil heaters wrapping the optical cylinder ensure a uniform temperature. An active regulation with a variable duty cycle is implemented in the LASCO/EIT control electronic. The thermal balance philosophy is to have a low equilibrium temperature (zero heater power dissipation) in the BOL, but not too low to be still able, using the dedicated power dissipation in the heater system to reach the required $20^{\circ} \mathrm{C}$. In the $\mathrm{EOL}$ warmest case, the design is trimmed to keep an equilibrium temperature (zero heater power dissipation) lower but close to $20^{\circ} \mathrm{C}$ with the adequate safety margins.

The design is such that in BOL conditions, the $20^{\circ} \mathrm{C}$ can be reached with less than $5 \mathrm{~W}$, assuming a zero power temperature $\mathrm{Tbol}^{*}=\mathrm{Tbol}$ - cold margins. In EOL conditions, the zero power temperature $\mathrm{Teol}^{*}=$ Teol + warm margins shall remain lower than $20^{\circ} \mathrm{C}$. Practically, the final design was defined with $\mathrm{Tbol}^{*} \sim 6^{\circ} \mathrm{C}$ and $\mathrm{Teol}^{*} \sim 17^{\circ} \mathrm{C}$.

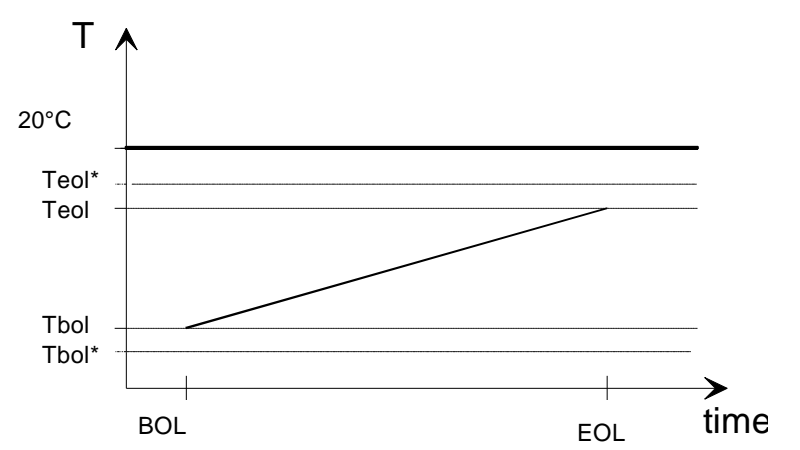

Figure (2.): Expected "zero power" temperature evolution with the instrument aging. 
The whole instrument is covered with a 20 layered thermal blanket (MLI) for radiative insulation. To meet all the thermal requirements on the optical system, a black anodized part of the structure is not covered by the MLI. Combined with a low heat sink temperature, this area can radiate exceeding power from the instrument. This uncovered area has been accurately trimmed after thermal tests at instrument level and final tests at S/C level, to limit the "zero power EOL temperature" to a value below $20^{\circ} \mathrm{C}$. This safety margin $\left(20^{\circ} \mathrm{C}\right.$ - "zero power" EOL temperature) includes uncertainties on the aging effect, computation inaccuracies, ... This choice of trimming the secondary radiator surface was preferred to our first idea of providing another trim capability e.g. by accommodating a pattern of different coatings in the predefined $\varepsilon / \alpha$ ratios, due to the difficulty to have stable thermo-optical properties (especially the solar absorbance) under permanent and perpendicular sun illumination.

\section{THERMAL BEHAVIOR OF THE ENTRANCE ALUMINUM FILTERS.}

An external optical front baffle is mounted on the front section of the instrument. It is a black cavity with a circular aperture defining the useful aperture entrance. The front section itself, inside the baffle, is completely obstructed by a wide aluminum filter, used to reject the visible light and the major part of the sun heat input.

This front filter, in four separate quarters, is made of $5 \mathrm{~mm} \times 5 \mathrm{~mm}$ Nickel grid and a celluloid layer encased between two very thin aluminum foils $(800 \AA$ each). The filter frames are mounted on the aluminum structure of the instrument, providing a good conductive path from the Ni grid to the structure.

Due to manufacturing process (vacuum deposition), the aluminum foils have a wrinkled aspect. The solar optical properties of these foils cannot be theoretically defined. The solar absorbed flux is a very important parameter driving the thermal behavior of the instrument. Thus specific tests were performed to characterize the solar reflected flux with a normal incidence. The angular distribution of the reflected flux gave the energy leaving the front baffle by the entrance aperture, and gave the flux distribution in the black cavity of the front part, in order to predict the solar absorbed flux.

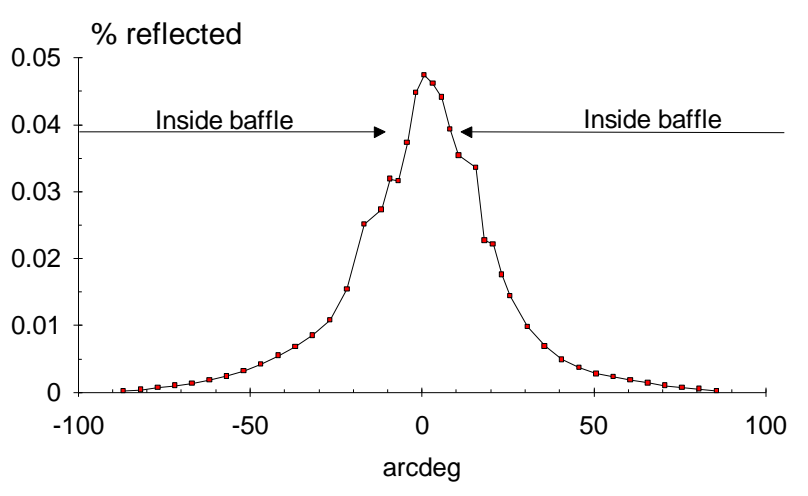

Figure (3.): Measured reflected flux under normal illumination.

The temperature distribution in the front section has been carefully studied by analyses and tests. The thermal behavior of the front part and its surroundings has also been evaluated by performing a specific test in the SIMLES solar simulator vacuum chamber at INTESPACE, with a numerous set of temperature sensors, after the S/C TB test.

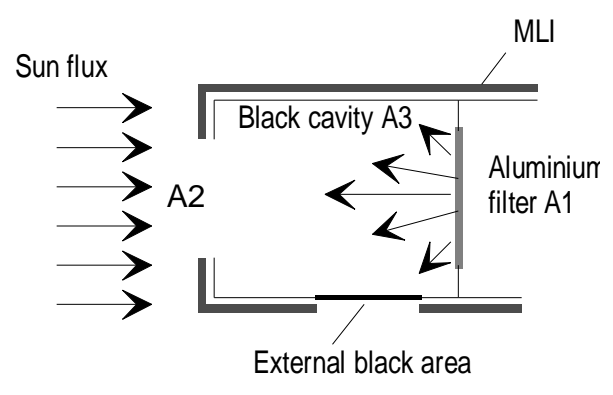

Figure (4.): Front section configuration.

The use of Gebhart factors, extended for a system exclusively composed of partly diffuse/partly specular, gray surfaces was very helpful to define the solar power absorbed by the different elements: the solar power absorbed by the filter is not simply: $F_{\text {solar }} \cdot A_{1} \cdot \alpha_{1}$ but should read $F_{\text {solar }} \cdot A_{1} \cdot\left[\alpha_{1}+\left(1-\alpha_{1}\right) \cdot B_{1,1}\right]$.

Entering solar power:

$$
F_{\text {solar }} \cdot A_{2}
$$

Leaving solar power:

$$
F_{\text {solar }} \cdot A_{2} \cdot\left(1-\alpha_{1}\right) \cdot B_{1,2}
$$

Solar power absorbed by the baffle:

$$
F_{\text {solar }} \cdot A_{2} \cdot\left[\left(1-\alpha_{1}\right) \cdot B_{1,3}\right]
$$

Solar power absorbed by the instrument front section (see annexes 2 and 3 for the definitions):

$$
F_{\text {solar }} \cdot A_{2} \cdot\left[1-\left(1-\alpha_{1}\right) \cdot B_{1,2}\right]
$$




$$
\begin{aligned}
& F_{1,1}^{s^{\prime}}=0 \\
& F_{1,2}^{s^{\prime}}=F_{1,2} \\
& F_{1,3}^{s^{\prime}}=F_{1,3} \\
& F_{2,2}^{s^{\prime}}=F_{2,(2,1)} \cdot \rho_{s, 1} \cdot\left(1-\rho_{s, 2}\right)=F_{2,(2,1)} \rho_{s, 1} \\
& F_{2,3}^{s^{\prime}}=\left[F_{2,3}+F_{2,(3,1)} \cdot \rho_{s, 1}\right]\left(1-\rho_{s, 3}\right)=F_{2,3}+F_{2,(3,1)} \rho_{s, 1} \\
& F_{3,3}^{s^{\prime}}=\left[F_{3,3}+F_{3,(3,1)} \cdot \rho_{s, 1}\right]\left(1-\rho_{s, 3}\right)=F_{3,3}+F_{3,(3,1)} \rho_{s, 1} \\
& A_{i}^{\prime} F_{i j}^{s^{\prime}}=A_{j}^{\prime} F_{j i}^{s^{\prime}} \text { or }\left(1-\rho_{s, i}\right) A_{i} F_{i j}^{s^{\prime}}=\left(1-\rho_{s, j}\right) A_{j} F_{j i}^{s^{\prime}} \\
& B_{i j}=F_{i j}^{s^{\prime}} \alpha_{j}^{\prime}+\sum_{\forall l_{d i f f u s e}} F_{i l}^{s^{\prime}}\left(1-\alpha_{l}^{\prime}\right) B_{l j}
\end{aligned}
$$

Another concern about the front filters was the local temperature of the aluminum foil, between the grid meshes. Hand calculations indicate a temperature higher than $100^{\circ} \mathrm{C}$, showing a potential damage to the filter when exposed to the sun. Thus a test was conducted at the ESA/ESTEC facilities with a flight representative front part facing a sun simulator. The aim of the test was to evaluate the solar absorbed flux, measure the filter temperature and monitor the temperature distribution in the front section. The ESA/ESTEC facility is equipped with a thermographic camera. A drop of black paint was initially deposited in the center of one aluminum cell of the filter back side. Its temperature was measured through a glass window of the vacuum chamber with the camera.

The resulting measurement was used to perform extensive thermal vacuum tests on filters. Their ability to withstand long exposure time at high temperature was demonstrated with optical stray-light measurements.

\section{THERMAL BEHAVIOR OF THE CCD DETECTOR.}

The detector of EIT is a specific EUV enhanced $1024 \times 1024$ pixel CCD, the housing of which is designed to be operated at very low temperatures. The CCD is mounted in the instrument at the location of the focal plane, at the rear of the primary mirror. The goal of the thermal design of the camera is to reach $-80^{\circ} \mathrm{C}$ at the level of the CCD chip. This thermal requirement is needed mainly for 2 reasons:

lower than $1 \mathrm{DN} / \mathrm{s} /$ pixel,

reduction of the dark current to values

reduction of the effects of the lattice defects produced by the radiations once in-orbit, the effects of photon traps being eliminated from the signal channel, improving the charge transfer efficiency.
Moreover, in order to outgass the accumulated contaminant on the CCD, a bake out heater is installed on the cold finger, allowing periodic warms up to $+15^{\circ} \mathrm{C}$.

The constrain on the available electrical resources did not allow the use of an active cooling system. Then a passive cooling by means of an external radiator has been implemented. The CCD chip is attached to an aluminum cold finger, extending itself outside the vacuum tight camera housing, and conductively connected to a radiating plate facing the cold space.

Reaching $-80^{\circ} \mathrm{C}$ was a difficult challenge from the thermal design point of view. The main difficulties can be listed as follow:

the read out electronics of the CCD has to be mounted close to the detector itself, providing a consequent unwanted heat source,

the camera housing is part of the overall vacuum tight vessel of the instrument. It shall provide vacuum feedthrough for the cold finger, providing also thermal leaks between the $+20^{\circ} \mathrm{C}$ housing and the conductive link of the CCD to the radiator,

the radiator, made of square aluminum plate, shall be firmly held by mechanical means to withstand the acoustic and random vibrations, adding then additional conductive thermal leaks

the view factor of the radiator toward the cold space has been reduced by the presence of warm S/C elements (increase of the heat sink temperature)

To face all these problems, the thermal design of the detector system has been carefully analyzed. We can enhance some specific relevant points.

Specific materials have been selected for their isolation/conductive properties, such as titanium and copper.

The radiator coating is an important parameter that has been selected to provide the highest heat rejection. A specific NASA GSFC yellow paint was chosen according to its high emissivity at $-90^{\circ} \mathrm{C}$.

conductances.

Indium is used to improve the contact

functions.

Isolators are also providing structural 


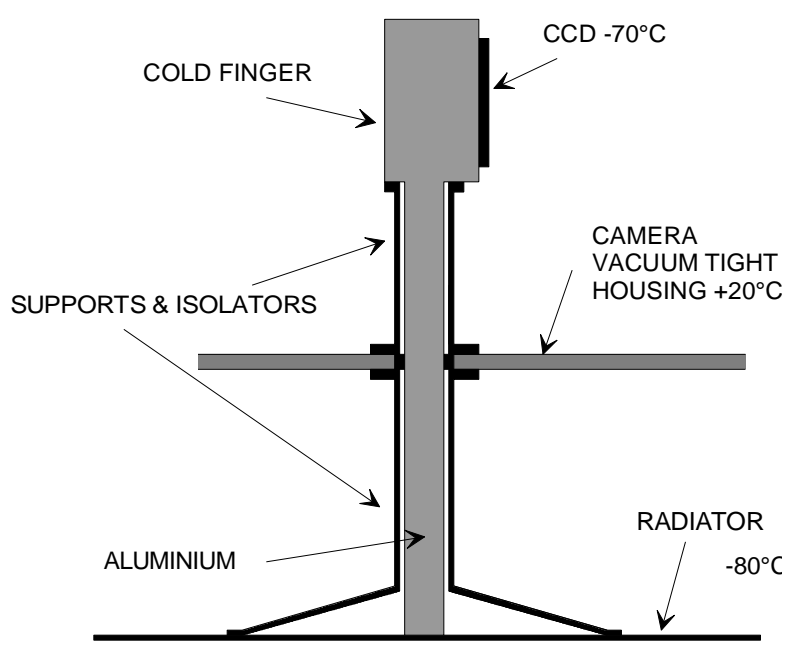

Figure (5.): CCD cooling system

Unfortunately the heat sink temperature increased with the development of the S/C. To cope with additional warm parts viewing the EIT radiator, a specific specular shield has been designed and finally mounted on the $\mathrm{S} / \mathrm{C}$ to hide these warm parts and decrease the heat sink temperature.

Specific tests at subsystem level (on the camera part) were conducted to evaluate the thermal flux within the cold finger during CCD operations. Then it allowed us to size the external radiator according to its heat sink temperature.

The S/C TB test did show a higher heat sink for the radiator, due to multiple solar reflections on S/C parts and grazing sun on the radiator itself due to the solar simulator divergence.

\section{SIMPLIFIED THERMAL TEST MATRIX OF THE EIT PFM}

\begin{tabular}{|l|c|c|c|}
\hline \multicolumn{1}{|c|}{ Elements } & $\begin{array}{c}\text { Subsystem } \\
\text { tests }\end{array}$ & $\begin{array}{c}\text { Instrument } \\
\text { test }\end{array}$ & $\begin{array}{c}\text { S/C } \\
\text { test }\end{array}$ \\
\hline $\begin{array}{l}\text { Optical } \\
\text { system }\end{array}$ & - & $\begin{array}{c}\text { TV, TB at } \\
\text { CSL }\end{array}$ & TB \\
\hline $\begin{array}{l}\text { Filter wheel } \\
\text { system }\end{array}$ & TV at LAS & $\begin{array}{c}\text { TV, TB at } \\
\text { CSL }\end{array}$ & TB \\
\hline $\begin{array}{l}\text { Rotating } \\
\text { mask }\end{array}$ & TV at LAS & $\begin{array}{c}\text { TV, TB at } \\
\text { CSL }\end{array}$ & TB \\
\hline Camera & TV, TB at NRL & $\begin{array}{c}\text { TV, TB at } \\
\text { CSL }\end{array}$ & TB \\
\hline $\begin{array}{l}\text { Aluminum } \\
\text { filter }\end{array}$ & $\begin{array}{c}\text { Specularity at PTS } \\
\text { TV at IAS } \\
\text { TB at ESTEC } \\
\text { TB in SIMLES }\end{array}$ & $\begin{array}{c}\text { TV, TB at } \\
\text { CSL }\end{array}$ & TB \\
\hline $\begin{array}{l}\text { Door } \\
\text { mechanism }\end{array}$ & $\begin{array}{c}\text { TV } \\
\text { TB at ESTEC }\end{array}$ & $\begin{array}{c}\text { TV, TB at } \\
\text { CSL }\end{array}$ & TB \\
\hline MLI & \multicolumn{1}{|c|}{ TB at CSL } & TB \\
\hline
\end{tabular}

\section{IN FLIGHT DIAGNOSTIC.}

The SOHO spacecraft was launched in December 95, and the final orbit was reached in Feb 96. The thermal behavior of EIT on SOHO is monitored thanks to several control thermistors and housekeeping sensors. After one year of operations and monitoring, we can highlight the following results:

1. The early monitoring of the CCD temperature, a few days after launch showed a quite high value, around $-50^{\circ} \mathrm{C}$. A significant decrease was recorded after a few weeks and several bake out cycles. The final equilibrium CCD temperature is close to $-70^{\circ} \mathrm{C}$. This improvement is attributed to the residual water vapor in the instrument, transformed into ice condensed on the coldest part, i.e. the cold finger linking the CCD to the radiator. This resulted in conductive heat leaks degrading the isolation of the cold finger from the warm housing. Pressure decrease combined with warm up of the cold finger allowed the release of this leak effect and improved the CCD radiator efficiency.

2. The S/C platform is correctly maintained around $+20^{\circ} \mathrm{C}$ thanks to the $\mathrm{S} / \mathrm{C}$ thermal control.

3. The optics are actively controlled to the nominal $+20^{\circ} \mathrm{C}$, and already provided tens of thousands of correctly focused images of the solar EUV emissions. The control coefficients of the thermal loop have been updated after a few weeks of observations, and ensure now a very stable internal environment $\left(<0.2^{\circ} \mathrm{C}\right)$, using fraction of the limited allocated power.

\section{NEEDED TOOLS}

In the development of a scientific payload, a complete and experimentally correlated thermal mathematical model is generally required. This complete thermal mathematical model (CTMM) is necessarily confronted with the experimental results of thermal balance tests or other more specific tests. These tests must be defined and the boundary conditions must be verified. Moreover, a reduced (or simplified) thermal mathematical model (RTMM) of the experiment, which is part of a larger payload or spacecraft, is generally requested by ESA to be integrated in a full thermal model of the complete spacecraft. What is presently done at ESA when a payload is composed of a large amount of experiments the responsibility of which is shared by several experimenters? 


\subsection{Experimenter's tasks}

Each experimenter must realize :

A. a complete thermal mathematical model (CTMM) of their experiment

B. a reduced thermal mathematical model (RTMM) of their experiment, "compatible" with the complete model

C. TB / TV / thermal identification tests to update the TMM and the RTMM.

D. Define the test conditions which simulate the space boundary (or simplified Space) conditions.

No tools are available to realize semiautomatically the tasks $B, C$ and $D$.

\subsection{ESA or Spacecraft's Contractor's tasks}

ESA has the following responsibilities:

A. build a complete thermal mathematical model of the spacecraft by using the RTMM of the experiments coming from the experimenters

B. define new boundary conditions for each experiment: these exchanges between ESA and experimenters are iterative (and we hope that it converges sufficiently rapidly; there is no well defined test for this).

C. ESA should also redefine the conditions of the TV tests after update of the TMM with TB tests.

\subsection{Tools to be developed}

1. Generate a reduced thermal mathematical model from the complete thermal mathematical model with some compatibility criteria to be agreed with ESA.

2. Define the test environment for given $\mathrm{T}_{\text {sink }}, \varepsilon_{\text {eff }}$ and for a given geometry of shrouds around the experiment.

3. Complete mathematical sensitivity analysis.

4. Definition of the tests (eventually at subsystem level) to be performed to identify the most sensitive, not well known parameters (MLI $\mathrm{k}_{\text {eff., }}$ contact conductance's, photon traps, sun shading effects, aging of thermo-optical properties...).

5. Correlation between tests and CTMM and RTMM in two steps and update of the CTMM and RTMM.

6. Generate the TV test conditions from the TB test results.

We are presently working to fill some lack in the available tools to have a quasi-automatic generation of a reduced model on one hand and to correlate and to update the thermal mathematical model in agreement with different test results on the other hand. The aim of our study is to define the methodology and afterwards the software

\section{DISCUSSION}

\subsection{TESTS AND CTMM CORRELATION AND CTMM UPDATE. METHODOLOGY AND TOOLS TO DEVELOPED}

In the development of a scientific payload, a complete thermal mathematical model is generally required. This complete thermal mathematical model is necessarily confronted with the experimental results of thermal balance tests. This study should cover the following points:

Identification of the badly identified parameters in the thermal model.

Sensitivity analysis of the different parameters and identification of the most sensitive parameters.

Determination without a complete calculation of the variation effects of different parameters: variation of the solar, aging of the thermal properties of the coatings and $\mathrm{MLI}, \ldots$.

Test definition to define with a better accuracy, the not well known and influent parameters. instrumentation.

Definition of the experimental

Tests / CTMM correlation and thermal mathematical model update using a semi-automatic update of the thermal mathematical model.

- Test data treatment in order to take into account the fact that the test data contains measurement errors and are less numerous than the numbers of nodes in the mathematical model. Some 
kind of projection of the experimental points on the theoretical nodes should be developed.

Definition of an "Objective" functional to stationarise: this objective function is a measure of the difference of the test results and the mathematical simulation taking into account the gap between the initial values (the physically most probable values) of the physical parameters and the values which optimizes the functional; the well known physical parameters don't appear in the functional definition.

\subsection{SENSITIVITY ANALYSIS}

The accuracy of the TMM can be evaluated with a sensitivity analysis, in which the key parameters affecting the TMM are identified with their uncertainties and the temperatures are obtained by varying one parameter at a time. The root of the sum of the squares of the $\Delta T$ with the nominal case, due to each parameter, gives the uncertainties at each nodes.

This sensitivity analysis can classify the parameters into global and local parameters according to a local or a general effect on the nodes. Global parameters have an effect on the mean nodes temperature while the local parameters have less. We will first optimize the cost function with respect to global parameters which are responsible for the general equilibrium of the experiment: these are the parameters responsible for the heat inputs and heat leaks: external thermo-optical properties, MLI $\mathrm{k}_{\text {eff }}$ and conduction through supports, solar absorptivity, solar input, other heat input. A RTMM aimed at reproducing the internal average temperatures may be useful at this stage for this first optimization.

Once the requirement on reproducing the internal average temperature is approached, a deep analysis of the local temperature distributions is needed and a new optimization on the CTMM, with respect to the only local parameters is now to be realized. These local parameters involve the understanding of contact conduction, the conductance through small structures (door hinges), the effects of items that are often not modeled like cables, tubes, waveguides, ... .

\subsection{ON THE TESTS DEDICATED TO SENSITIVE SUBSYSTEMS}

In many thermal designs there remains some uncertainties that need to be evaluated before correlating the final thermal model. From the experience gained during the EIT program, we emphasize the future development teams to identify the potential problem areas and to implement specific verification programs at subsystem level. Confidence gained by these additional verifications will always be fruitful to assess the thermal design.

\subsection{ON THE INSTRUMENT TB TEST.}

\subsubsection{Test definition}

When the experiments are well isolated and when the problem is mainly radiative (i.e. linear in $\mathrm{T}^{4}$ ), (This is the case on SOHO where the heat exchanges by radiation and conduction are limited in the EID-A), the thermal environment of an instrument on the Spacecraft can be simulated by a sink temperature and an effective emissivity for each external surface.

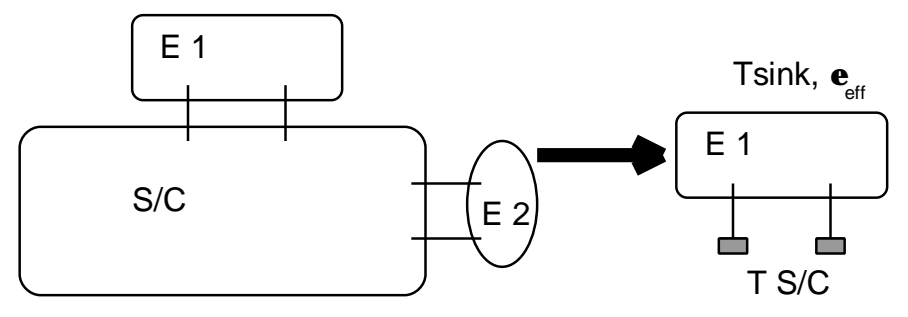

Fig. (6.) Definition of the "instrument" thermal boundary conditions

Tsink is the equilibrium temperature of an external surface of the experiment when it is isolated from inside (no power passing this surface), $\varepsilon_{\text {effective }}$ represents the radiative link when a thermal flow is allowed. This procedure is well known in the electrical circuit theory and corresponds to the Thevenin - Norton Theorem. It can even be generalized to more than one extracted node as shown below.

When there is no solar or other "visible" light absorbed by the surface, Tsink is not dependent on the thermo-optical properties of the considered surface but when there is a solar or another "visible" wave absorbed, the sink temperature strongly depends on the thermo-optical properties of the given surface and this can lead to an incorrectly evaluated sink temperature.

This notion of Tsink, $\varepsilon_{\text {effective becomes }}$ crumbling when one external experiment surface (like a radiator ) has a large view factor with another surface of the same experiment; this gives errors in the temperature of the radiator and of the detector connected to it !!. This is nevertheless the method applied to the SOHO project (and other projects).

In such situations, we should use the ThéveninNorton theorem in the matrix form:

\subsubsection{Extension of the $\mathrm{T}_{\text {sink, }}, \underline{\varepsilon_{\text {effective }}} \underline{\text { method. }}$}

The temperatures of all the external surfaces of the payload are separated into two vectors: one vector containing the external nodes of the experiment and the other vector, the nodes of the rest of the payload, denoted S/C: 


$$
\begin{aligned}
& \vec{U}_{\exp }=\left(\sigma \cdot T_{\exp }^{4}\right) \\
& \vec{U}_{S / C}=\left(\sigma \cdot T_{S / C}^{4}\right) \\
& \vec{U}_{\exp } \text { in } \mathrm{W} / \mathrm{m}^{2}
\end{aligned}
$$

$\vec{I}_{\text {exp }}$ is the power (W) dissipated by each surface of the experiment; $Z_{i j}$ is the impedance, the radiative coupling or the Gebhard coupling $\left(\mathrm{m}^{-2}\right)$. So we can write:

$$
\left(\begin{array}{l}
\vec{U}_{\text {exp }} \\
\vec{U}_{S / C}
\end{array}\right)=\left[\begin{array}{ll}
\underline{Z_{e e}} & \underline{\underline{Z_{e s}}} \\
\underline{\underline{Z_{s s}}}
\end{array}\right] \cdot\left(\begin{array}{l}
\vec{I}_{\text {exp }} \\
\vec{I}_{S / C}
\end{array}\right)
$$

\section{THEVENIN}

$$
\begin{aligned}
& \vec{U}_{\exp }=\vec{E}_{T h}+\underline{\underline{Z_{T h}}} \cdot \vec{I}_{\exp } \\
& \vec{E}_{T h}=\underline{\underline{Z_{e s}}} \cdot \underline{\underline{Z_{s s}^{-1}}} \cdot \vec{U}_{S / C} \\
& \underline{\underline{Z_{T h}}}=\underline{\underline{Z_{e e}}}-\underline{\underline{Z_{e s}}} \cdot \underline{\underline{Z_{s s}}} \cdot \underline{\underline{Z_{s e}}}
\end{aligned}
$$

This leads to the following equivalent graph when we reduce the problem to one surface at a time:

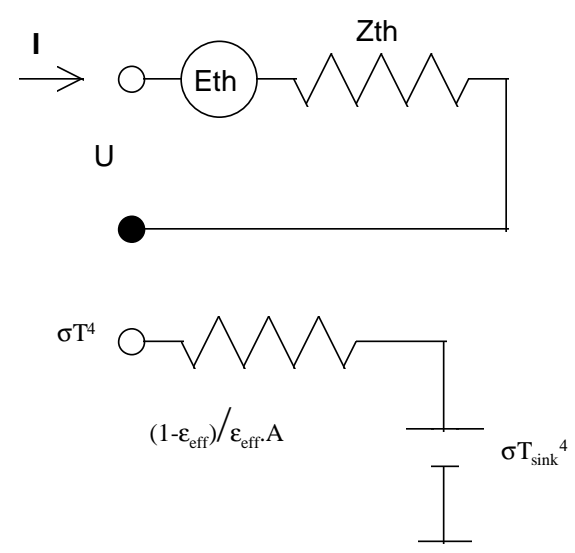

Fig. (7.) Equivalent Thevenin boundary condition for each external surface
NORTON

$$
\begin{aligned}
& {\left[\begin{array}{ll}
Z_{e e} & \underline{Z_{e s}} \\
\overline{\overline{Z_{s e}}} & \underline{\underline{Z_{s s}}}
\end{array}\right]^{-1}=\left[\begin{array}{ll}
\underline{Y_{e e}} & \underline{\underline{Y_{e s}}} \\
\overline{\underline{Y_{s e}}} & \underline{\underline{Y_{s s}}}
\end{array}\right]} \\
& {\left[\begin{array}{ll}
\underline{\underline{Y_{e e}}} & \underline{\underline{Y_{e s}}} \\
\underline{\underline{Y_{s e}}} & \underline{\underline{Y_{s s}}}
\end{array}\right]_{i j}=\varepsilon_{i} A_{i} \delta_{i j}-Y_{i j}} \\
& \vec{I}_{\exp }=\vec{I}_{N t}+\underline{\underline{Y_{N t}}} \cdot \vec{U}_{\exp } \\
& \vec{I}_{N t}=-\underline{\underline{Y_{e s}}} \cdot \underline{\underline{Y_{s s}^{-1}}} \cdot \vec{I}_{S / C} \\
& Y_{N t}=\underline{\underline{Y_{e e}}}-\underline{\underline{Y_{e s}}} \cdot \underline{\underline{Y_{s s}^{-1}}} \cdot \underline{\underline{Y_{s e}}}
\end{aligned}
$$

Where $Y_{i j}, \delta_{i j}$ represent the radiosity coupling and the Kronecker symbol.

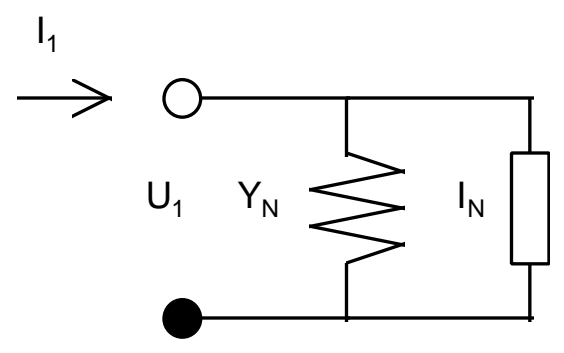

Fig. (8.) Equivalent Norton boundary condition for each external surface

When 2 surfaces of the same experiment are strongly radiatively coupled, it is better to use the following approximation:

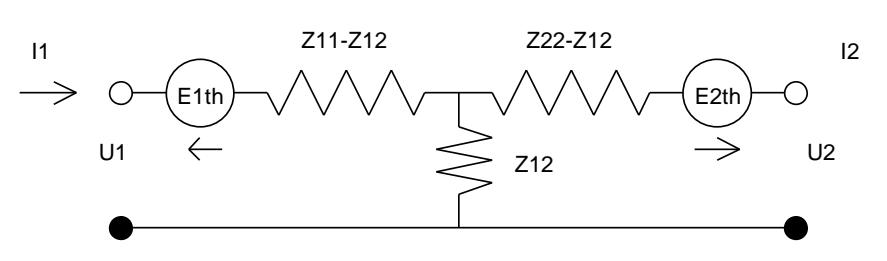

Fig. (9.) Equivalent Thevenin Norton boundary condition for two radiatively coupled external surfaces

\subsubsection{TB tests at experimenters side}

Since it is not feasible to simulate the S/C. at instrument level TB test, we simulate the following boundary conditions:

- $\quad \mathrm{T}_{\text {sink }}, \varepsilon_{\text {eff. }}$ for the external surfaces 
- $\quad$ Solar heat input

- $\quad$ Other "visible " heat input.

How can we realize this TB test?

In theory, we simply could do the following:

- Use a sun simulator or simulate the solar heat input or compute the equivalent $\mathrm{T}_{\text {sink }}, \varepsilon_{\text {eff }}$ (which in this special case are coating dependent !)

- $\quad$ use a shroud very close to its corresponding surface and impose on this shroud $\mathrm{T}_{\text {sink }}$, $\varepsilon_{\text {eff. }}$

\section{Problem}

It is not practically feasible to obtain the good view factors $F_{i, s i}=1$ and $F_{i, s j}=0$ where i represents the experiment external surface $\mathrm{i}$ and $\mathrm{si}$, the corresponding shroud.

So we must change the temperatures of the shrouds to obtain an equivalent environment:

$$
\left(\begin{array}{c}
\vec{U}_{\text {exp }} \\
\vec{U}_{\text {Shrouds }}
\end{array}\right)=\left[\begin{array}{ll}
\frac{Z_{e, e}}{\underline{\overline{Z_{e, s}}}} & \overline{\underline{Z_{s, e}}}
\end{array}\right] *\left(\begin{array}{l}
\vec{I}_{\text {exp }} \\
\vec{I}_{\text {Shr }}
\end{array}\right)
$$

The number of shrouds is equal to the number of external surfaces of the RTMM $=\mathrm{n}$, so $\left[\begin{array}{ll}\frac{Z_{e, e}}{\underline{\underline{Z_{s, e}}}} & \underline{\underline{\underline{Z_{s, s}}}}\end{array}\right]$ are 4 matrices $(n \times n)$.

Therefore we can impose:

$$
\begin{aligned}
\vec{E}_{\text {Thev, test }} & =\underline{\underline{Z_{e, s}}} \cdot \underline{\underline{Z^{-1}{ }_{s, s}}} \cdot \vec{U}_{S} \\
& =\overrightarrow{\mathrm{E}}_{\text {Thev, ESA }} \text { corresponding to the sink }
\end{aligned}
$$

temperatures imposed by ESA.

So we obtain the temperatures to be applied to the shrouds:

and

$$
\vec{U}_{S}=\underline{\underline{Z_{s, s}}} \cdot \underline{\underline{Z^{-1}{ }_{e, s}}} \cdot \overrightarrow{\mathrm{E}}_{\text {Thev, ESA }}
$$

$$
\begin{aligned}
\operatorname{diag}\left(\underline{\underline{Z_{\text {Thev }}}}\right) & =\operatorname{diag}\left(\underline{\underline{Z_{e, e}}}-\underline{\underline{Z_{e, s}}} \cdot \underline{\underline{Z^{-1}}} \underline{\underline{\underline{Z_{\text {thev }, E S A}}}}\right) \\
& =\operatorname{diag})
\end{aligned}
$$

In fact, when $F_{i, s i}<1$, the test is not $100 \%$ representative due to the following situation:

ESA imposes equations:

$$
\begin{aligned}
& U_{1}=E_{1}+Z_{1,1} \cdot I_{1} \\
& U_{2}=E_{2}+Z_{2,2} \cdot I_{2} \\
& \ldots \\
& U_{n}=E_{n}+Z_{n, n} . I_{n}
\end{aligned}
$$

Experimenter tests:

$$
\begin{aligned}
& U_{1}=E_{1}+Z_{1,1} \cdot I_{1}+Z_{1,2} \cdot I_{2}+\ldots+Z_{1, n} \cdot I_{n} \\
& U_{2}=E_{2}+Z_{2,1} \cdot I_{1}+Z_{2,2} \cdot I_{2}+\ldots+Z_{2, n} \cdot I_{n} \\
& \ldots \\
& U_{n}=E_{n}+Z_{n, 1} \cdot I_{1}+Z_{n, 2} \cdot I_{2}+\ldots+Z_{n, n} \cdot I_{n}
\end{aligned}
$$

In the future ESA could furnish the complete system of equations.

\subsubsection{EIT Thermal balance test}

A thermal balance test at instrument level was conducted before delivery to the S/C. The ESA heat sink temperature were used to define the 6 shroud envelope thermal environment. The sun effect was simulated by a warm black shroud, heated to provide an equivalent environment and solar absorbed flux.

In this philosophy, it is assumed that the solar absorptivity of the sun exposed surfaces is well known, in order to be flight representative of the sun heat input to the instrument. Further tests revealed that the sun input obtained during this TB test was not flight representative at all. The effective characterization of the sun input required additional tests with sun simulator at subsystem level, due to critical areas in the EIT front section.

Simulating the sun by a warm black plate is an approach that shall be used with a lot of care, as it requires a prerequisite good knowledge of the thermooptical properties of the instrument surfaces.

\subsection{ON THE S/C TB TEST.}

The EIT proto-flight instrument did see the sun during vacuum tests only at $\mathrm{S} / \mathrm{C}$ level. This test at a late stage in the project allowed us to perform a good correlation with the thermal models. Such a good correlation was not possible with the TB test at instrument level without solar simulator, because of all the specific effects of the sun, like additional fluxes on the appendixes, solar traps in the front baffle, reflections on coatings and on front aluminum filters, ...

Like EIT, most of the instruments of SOHO encountered discrepancies between the tests and their mathematical 
model. The overall discrepancy was a combination of two errors. One was related to the test environment. After the test, the test chamber was discovered to be incorrectly modeled thus contributing to a higher IR sink temperature. In addition, the solar simulator provided between 6.5 and $9.5 \%$ more flux (depending where the instrument was located) than the one specified. The second error included modeling, workmanship and other errors made either by the system or the experimenters at several stages (model or hardware). After the S/C TB test, the correlation activity was performed in parallel: the prime contractor was responsible to correlate the SVM, the PLM and the instrument electronics. The experimenters were responsible to "re-correlate" their respective instruments. This was made possible because the sensors thermal design was rather independent from the S/C. After being correlated, the instrument models have been incorporated into the overall S/C model for a new round of analyses. For the second time, the measured temperatures have been compared to the predictions. In order to better correlate the test data, most of the instruments had to adjust thermal parameters in their TMM. Few instruments, CDS, MTOF, EIT and MDI modified the hardware before the flight. EIT modified the coating of its door to make it cooler. Once the modifications/adjustments of the mathematical models, and/or flight hardware, another run of the S/C model in the flight environment has been done.

The reasons of the errors are numerous. We can classify the instruments into 3 categories as follows.

In the first category fall the instruments which did not need to perform any correlation after the S/C TB test. It was the case of LION, SWANI, SWAN2 and VIRGO.

The second category gathers the instruments which did not correlate because of spurious effects of the sun like additional fluxes on appendices, solar trapping, reflections on coatings.... It was the case of CDS, EIT, MDI and to a certain extent UVCS. Today, we believe that without the solar simulation during the system TB Test, the flight performance of these instruments would have been seriously jeopardized.

In the last category fall the instruments which did not correlate for other reasons than those given above. It can be because of the gradients, the MLI efficiency, early instrument TB test.

Overall, it is clear that the S/C TB test did bring a lot to the experiments and avoided some instruments to have a bad surprise in flight. Moreover, the presence of the sun during the S/C TB test did bring additional information on the instrument thermal behavior. We have tried to list here below the advantages of a solar simulation test at system level.
- $\quad$ to avoid problems generated by early instrument TB test where the instrument was not fully representative of the flight model in terms of thermo-optical properties, appendices, MLI efficiency, power dissipation...

- $\quad$ to get the correct MLI temperatures and induced environment in cold areas where cold radiators are located.

- $\quad$ to uncover workmanship errors e.g. wrong thermal finishes (measurement before the test is not always feasible (time, cost...), solar trapping, MLI shaped differently...

- $\quad$ to get the MLI efficiency where the MLI is hit by the sunlight for instruments sensitive to the MLI efficiency.

- $\quad$ to uncover modeling errors because of the coarseness of the TMM e.g. complex shapes simplified (appendices like door motors, small shield, external cables), apertures and baffles treatment, size of the external nodes in cold radiator areas.

- $\quad$ to uncover spurious reflections that may affect the performance of another instrument detector but do not affect the instrument thermal behavior.

The S/C test was very useful for EIT to cope with all these sun effects, combined with the presence of other S/C parts. As a recommendation, we suggest to all the instruments exposed to the sun during flight operations, to be vacuum tested in front of a solar simulator at least once. If the sun simulator is used only once, it is preferable to use it at Spacecraft level tests due to additional possible interaction / interference from one instrument to another. This recommendation is purely based on technical arguments.

From a programmatic point of view, it might be desirable to perform the instrument solar simulation but earlier than the system TB test. Provided that the build standard of the instrument to be tested is similar to the FM, the test will help at identifying potential hardware updates as early as possible in the program.

\section{CONCLUSIONS}

Despite a lot of critical areas, the EIT program is a complete success.

From the thermoelastic point of view, no decentring is recorded, demonstrating the good behavior of both the S/C and the EIT structures. This can be attributed to a good S/C thermal design, an adequate quasi-isostatic mounting concept and good concept of the EIT internal structure. 
The thermal status of the instrument under the real operational environment is also fully compliant with the pre-flight predictions. The optical system temperature is the most critical point, as the image focus is directly linked to it. The operational situation gives full satisfaction in term of the achieved thermal focusing, demonstrating the adequacy of the thermal model used to size the radiator area on the front baffle and to achieve a thermal control with the allocated limited power.

We can only regret the high CCD radiator temperature, reaching the higher acceptable limit of the CCD operational temperature $\left(-70^{\circ} \mathrm{C}\right)$. This warmer environment can be attributed to several factors, contributing all to a higher radiator heat sink temperature:

- unexpected incident solar flux (direct or indirect sources) on the radiator plate itself. This could be avoided be adding at the baffle level a sun shield which puts the radiator in the shadow of the sun.

- unexpected incident solar flux (direct or indirect sources) on the additional sun shield

- warmer S/C parts in the view angle of the radiator

\section{ACKNOWLEDGMENTS}

EIT is the result of the combined efforts of an international consortium, involving Belgian institutes (CSL, ORB), French laboratories (IAS, IOTA and LAS) and American laboratories (NRL, LM, NASA GSFC).

The Belgian institutes were funded by SSTC, via PRODEX. The French laboratories were supported by CNRS and CNES. The American groups received support from the Office of Naval Research and from NASA. We acknowledge the PTS GMBH (Germany) for producing the EIT filters and providing measurements used in this paper.

\section{REFERENCES}

[1] The SOHO mission, Scientific and Technical Aspects of the Instruments, ESA SP-1104, 1988

[2] Design and qualification of the Extreme-UV Imaging Telescope, JM. Defise, P. Rochus. IAF-95U.4.08.

[3] A thermal analysis approach applied in early stages of a program, G.W. Dierssen, Proceedings of the International Symposium on Environmental and Thermal Systems for Space Vehicles, Toulouse , 4-7 Oct. 1983. (ESA SP-200, Dec 1983).
[4] EIT: Extreme-Ultraviolet Imaging Telescope for the SOHO mission, J.M.Defise, C. Jamar, P. Rochus et al. ; Solar Physics 162; 291-312, 1995

[5] Radiation heat transfer at a surface having both specular and diffuse reflectance components. E.M. Sparrow and S.L. Lin; Int. J. Heat Mass Transfer. Vol.8 (1965) pp. 769-779.

\section{ADDITIONAL SOURCES}

PTS. Technical note.

Poinas P. Personal communication.

\section{DEFINITIONS, ACRONYMS, ABBREVIATIONS}

$\begin{array}{ll}\text { BOL } & \text { Beginning Of Life } \\ \text { CCD } & \text { Charge Coupling Device } \\ \text { CSL } & \text { Centre Spatial de Liège } \\ \text { DN } & \text { Digit Number } \\ \text { EID-A } & \text { Experiment Interface Document - Part A } \\ \text { EIT } & \text { Extreme-UV Imaging Telescope } \\ \text { EOL } & \text { End Of Life } \\ \text { ESA } & \text { European Space Agency } \\ \text { ESTEC } & \text { European Space Technology Centre } \\ \text { EUV } & \text { Extreme-Ultraviolet } \\ \text { GSFC } & \text { Goddard Space Flight Centre } \\ \text { IAS } & \text { Institut d'Astrophysique Spatiale } \\ \text { IOTA } & \text { Institut d'Optique Théorique Appliquée } \\ \text { LAS } & \text { Laboratoire d'Astronomie Spatiale } \\ \text { LASCO } & \text { Large Angle Spectroscopic } \\ & \text { Coronagraph } \\ \text { LM } & \text { Lockheed Martin } \\ \text { MLI } & \text { Multilayer Insulation } \\ \text { NRL } & \text { Naval Research Laboratory } \\ \text { ORB } & \text { Observatoire Royal de Belgique } \\ \text { PFM } & \text { Proto-Flight Model } \\ \text { PTS } & \text { Physkalisch-Technische Studien } \\ \text { S/C } & \text { Spacecraft } \\ \text { SIMLES } & \text { Simulateur d'Environnement Spatial } \\ \text { SOHO } & \text { Solar and Heliospheric Observatory } \\ \text { TB } & \text { Thermal Balance } \\ \text { TV } & \text { Thermal Vacuum }\end{array}$




\section{ANNEX 1}

\section{Definition of the Gebhart absorption factors $B_{i, j}$ and the radiosities $Y_{i, j}$}

The GEBHART absorption factor $\mathrm{B}_{\mathrm{i}, \mathrm{j}}$ (nondimensional quantity) is the proportion of the energy diffusely emitted by surface $\mathrm{i}$ (by its temperature), which finally after multiple reflections by all the surrounding surfaces, is absorbed by the surface $\mathrm{j}$. $\mathrm{B}_{\mathrm{i}, \mathrm{i}}$ is generally not naught.

The net radiation exchange between surface $\mathrm{i}$ and surface j reads:

$$
q_{i \rightarrow j}=\varepsilon_{i} \cdot A_{i} \cdot E_{b}\left(T_{i}\right) \cdot B_{i, j}-\varepsilon_{j} \cdot A_{j} \cdot E_{b}\left(T_{j}\right) \cdot B_{j, i}
$$

where $E_{b}(T)$ is the black-body radiation.

\section{General properties of the Gebhart factors}

1) $\quad \sum_{\forall j} B_{i, j}=1$

which means that the energy emitted by surface $\mathrm{i}$, is finally absorbed by one of the surrounding surfaces. To avoid the possibility of a ray escaping the system to infinity, we add to the existing surfaces, a black-body surface surrounding the complete system.

2)

$$
\varepsilon_{i} \cdot A_{i} \cdot B_{i, j}=\varepsilon_{j} \cdot A_{j} \cdot B_{j, i}
$$

In fact by definition of $B_{i, j}$, we obtain

$$
\begin{aligned}
q_{i \rightarrow j} & =\varepsilon_{i} \cdot A_{i} \cdot E_{b}\left(T_{i}\right) \cdot B_{i, j}-\varepsilon_{j} \cdot A_{j} \cdot E_{b}\left(T_{j}\right) \cdot B_{j, i} \\
& =\varepsilon_{\mathrm{i}} \cdot A_{i} \cdot E_{b}(T) \cdot B_{i, j}-\varepsilon_{j} \cdot A_{j} \cdot E_{b}(T) \cdot B_{j, i} \\
& =\left[\varepsilon_{\mathrm{i}} \cdot A_{i} \cdot B_{i, j}-\varepsilon_{j} \cdot A_{j} \cdot B_{j, i}\right] \cdot E_{b}(T)
\end{aligned}
$$

when $T=T_{i}=T_{j}$

If only the surfaces $i$ and $j$ are emitting energy, due to their temperatures, the other surfaces playing the role of simple reflectors, then when $T_{i}=T_{j}$, the net exchange between $\mathrm{i}$ and $\mathrm{j}$ should be zero, so we have:

$$
\varepsilon_{i} A_{i} B_{i, j}=\varepsilon_{j} A_{j} B_{j, i}
$$

(without making any assumption for the reflectivities of the different surfaces).

Therefore in the most general case, the heat exchange reads:

$$
\begin{aligned}
q_{i \rightarrow j} & =\varepsilon_{i} \cdot A_{i} \cdot E_{b}\left(T_{i}\right) \cdot B_{i, j}-\varepsilon_{j} \cdot A_{j} \cdot E_{b}\left(T_{j}\right) \cdot B_{j, i} \\
& =Y_{i, j} \cdot\left\{E_{b}\left(T_{i}\right)-E_{j}\left(T_{j}\right)\right\}
\end{aligned}
$$

where $Y_{i, j}=\varepsilon_{i} \cdot A_{i} \cdot B_{i, j}$ is the radiosity (for any type of reflection).
Equation for the Gebhart factors when all the surfaces are gray and reflecting diffusely

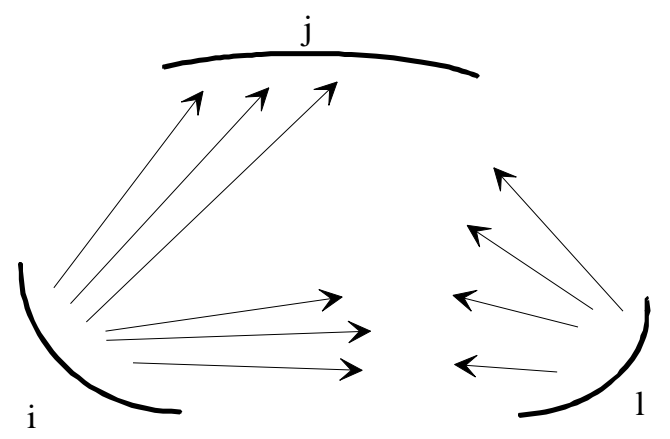

Figure (10.): Surfaces $\mathrm{i}, \mathrm{j}, 1$ gray and reflecting diffusely

The purpose of this paragraph is to obtain an equation for the Gebhart factor when all the surfaces satisfy the following assumptions which are usual for hand calculations:

1. each surface is isothermal

2. each surface is a gray body

3. each surface is a diffuse emitter

4. each surface is a diffuse reflector

5. the radiosity is uniformly distributed across each surface

The energy leaving $\mathrm{i}$ and absorbed by $\mathrm{j}$ can be separated in the following contributions:

- the energy directly emitted to $\mathrm{j}$ and absorbed by $\mathrm{j}$.

- the energy which is first diffusely reflected by one of the surfaces.

The beam coming from i and reflected by 1 , is diffusely reflected so there is no memory of the incoming beam; the proportion of this diffusely reflected beam which is finally absorbed by $\mathrm{j}$ is similar to the proportion of energy emitted by the surface 1 , and absorbed by $\mathrm{j}$ : i.e. $B_{l, j}$.

So we can write:

$$
B_{i, j}=F_{i, j} \cdot \varepsilon_{j}+\sum_{\forall l} F_{i, l} \cdot\left(1-\varepsilon_{l}\right) \cdot B_{l, j}
$$

where $F_{i, j}$ represents the view factor and $1-\varepsilon_{l}=\rho_{d, l}$ is the diffuse reflectivity of surface 1 .

Or in a matrix form :

$$
\underline{\underline{B}}=\underline{\underline{\beta}}+(\underline{\underline{F}}-\underline{\underline{\beta}}) \cdot \underline{\underline{B}}
$$

(when all the reflections are diffuse), where $\beta_{i, j}=F_{i, j} . \varepsilon_{j}$

Demonstration of the general properties of the Gebhart factors in the case of diffuse reflections

Our aim is to redemonstrate the two general properties of the Gebhart factors: 


$$
\varepsilon_{i} \cdot A_{i} \cdot B_{i, j}=\varepsilon_{j} \cdot A_{j} \cdot B_{j, i}
$$

in the case of diffuse reflections, making use of the equation of $B_{i, j}$ :

$$
\underline{\underline{B}}=\underline{\underline{\beta}}+(\underline{\underline{F}}-\underline{\underline{\beta}}) \cdot \underline{\underline{B}}
$$

A)

$$
\begin{aligned}
\varepsilon_{i} \cdot A_{i} \cdot B_{i, j}= & \varepsilon_{i} \cdot A_{i} \cdot F_{i, j} \cdot \varepsilon_{j} \\
& +\sum_{l} \varepsilon_{i} \cdot A_{i} \cdot F_{i, l} \cdot \varepsilon_{l}\left(\frac{1-\varepsilon_{l}}{\varepsilon_{l}}\right) \frac{\varepsilon_{l} \cdot A_{l}}{\varepsilon_{l} \cdot A_{l}} B_{l, j}
\end{aligned}
$$

or $\quad \underline{\underline{Y}}=\underline{\underline{U}}+\underline{\underline{U}} \cdot \underline{\underline{G}} \cdot \underline{\underline{Y}}$

where $U_{i, j}=\varepsilon_{i} \cdot A_{i} \cdot F_{i, j} \cdot \varepsilon_{j}=U_{j, i}$

and $\quad \underline{\underline{G}}=\operatorname{diag}\left(\frac{1-\varepsilon}{\varepsilon^{2} \cdot A}\right)$.

$$
\underline{\underline{U}}=\underline{\underline{U}}^{T} ; \underline{\underline{G}}=\underline{\underline{G}}^{T} \Rightarrow \underline{\underline{Y}}=\underline{\underline{Y}}^{T}
$$

since $\quad \underline{\underline{Y}}=\underline{\underline{U}}+\underline{\underline{U}} \underline{\underline{G}} \underline{\underline{U}}+\underline{\underline{U}} \underline{\underline{G}} \underline{\underline{\underline{G}}} \underline{\underline{\underline{U}}}+\cdots$

where each term is symmetrical so Property 2 is demonstrated.

\section{B) Property 1:}

$$
\begin{aligned}
& \sum_{\forall_{j}} B_{i, j}=1 ? \\
& \text { or } \sum_{j} Y_{i, j}=\varepsilon_{i} \cdot A_{i}, \text { or } \underline{B} \cdot \underline{u}=\underline{u} ?
\end{aligned}
$$

where the vector $\underline{u}$ is defined by $u_{i}=1$ for $\forall \mathrm{i}$.

The view factors satisfy the equation: $\underline{F} \cdot \underline{u}=\underline{u}$ (or $\sum_{j} F_{i, j}=1$ for $\forall \mathrm{i}$ ).

$$
\begin{aligned}
& \underline{\underline{B}}=\underline{\underline{[I-F+\beta]^{-1}}} \cdot \underline{\underline{\beta}} \\
& \underline{\underline{\beta}}=\underline{\underline{\overline{[I-F+\beta]}}}-\underline{\underline{[I-F]}}
\end{aligned}
$$

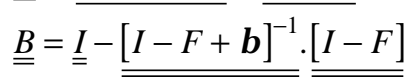

$$
\begin{aligned}
& \underline{\underline{B}} \underline{u}=\underline{\underline{u}}-\underline{\underline{[1-F+\beta]^{-1}}} \cdot[\underbrace{\underline{\underline{u}-\underline{\underline{F}}} \underline{u}}_{=0}]
\end{aligned}
$$

so $\quad \underline{B} \underline{\underline{u}}=\underline{u}$

\section{Remark:}

If we try to solve the equation $\underline{\underline{B}}=\underline{\underline{\beta}}+(\underline{\underline{F}}-\underline{\underline{\beta}}) \cdot \underline{\underline{B}}$ with an iterative method (i.e. perturbations method), the approximated solution will never satisfy the relation $\underline{\underline{B}} \underline{\underline{u}}=\underline{u}$

$$
\begin{aligned}
& \underline{\underline{B}}=\underline{\underline{\beta}}+(\underline{\underline{F}}-\underline{\underline{\beta}}) \underline{\underline{B}} \\
& \cong\left[\underline{\underline{\beta}}+(\underline{\underline{F}}-\underline{\underline{\beta}}) \cdot \underline{\underline{\beta}}+\left(\underline{\left.\underline{F}-\underline{\beta})^{2} \cdot \underline{\underline{\beta}}+\cdots\right]}\right.\right. \\
& \underline{\underline{B}} \cdot \underline{\underline{u}} \cong\left[\left[\underline{\underline{\beta}}+(\underline{\underline{F}}-\underline{\underline{\beta}}) \cdot \underline{\underline{\beta}}+\left(\underline{\left.\underline{F}-\underline{\underline{\beta}})^{2} \cdot \underline{\underline{\beta}}+\cdots\right] \underline{u}}\right.\right.\right. \\
& \quad \neq \underline{u} \text { at any order of perturbation. }
\end{aligned}
$$

This also explains why in iterative numerical methods, the calculated "radiosity" matrix doesn't satisfy $\sum_{j} Y_{i, j}=\varepsilon_{i} . A_{i}$; moreover, the symmetry $\underline{Y}=\underline{Y}^{T}$ is often not respected in numerical approximations and must be artificially symmetrised.

\section{ANNEX 2: DEFINITION OF VIEW FACTORS AMONG NON-LAMBERTIAN SURFACES (IN PRESENCE OF PARTLY SPECULAR / PARTLY DIFFUSE SURFACES)}

The purpose of this paragraph is to generalize the notion of view factor and to define the specular view factor, when all the surfaces satisfy the usual assumptions which allows hand calculations (ref 5):

1. each surface is isothermal

2. each surface is a gray body

3. each surface is a diffuse emitter

4. each surface is a diffuse reflector

5. the radiosity is uniformly distributed across each surface

except 4 which is relaxed and replaced by a more general condition:

4'. each surface is a partly diffuse / partly specular reflector.

So we want to extend the analysis of view factors and Gebhart factors to take into account some simple geometry's containing surfaces that emit radiation diffusely but that may reflect radiation partly in a specular manner and partly in a diffuse manner.

For each surface, we take the reflectivity to be the sum of a specular component and a diffuse component:

$$
\begin{aligned}
& \rho_{d}+\rho_{s}+\alpha=1 \\
& \alpha=\varepsilon
\end{aligned}
$$

as it is assumed that global Kirchhoff's identity still applies.

$G$ represents the heat irradiation of the surface (total radiation energy incident upon a surface per unit time and per unit area), $E_{b}$ the black-body radiation, $J_{d}$ the diffuse radiosity of the surface (total radiation energy which leaves a surface per unit time and per unit area); so the net diffuse heat exchange and the diffuse radiosity read:

$$
\begin{aligned}
& q=A \cdot\left(\varepsilon \cdot E_{b}-\alpha \cdot G\right) \\
& J_{d}=\left(\varepsilon \cdot E_{b}+\rho_{d} . G\right) \text { or } G=\frac{1}{\rho_{d}} \cdot\left(J_{d}-\varepsilon \cdot E_{b}\right)
\end{aligned}
$$




$$
\begin{aligned}
q & =A \cdot\left[\varepsilon \cdot E_{b}-\frac{\alpha}{\rho_{d}} \cdot\left(J_{d}-\varepsilon \cdot E_{b}\right)\right] \\
& =\frac{\varepsilon \cdot A}{\rho_{d}} \cdot\left[E_{b} \cdot\left(1-\rho_{s}\right)-J_{d}\right] \\
q & =\frac{E_{b}-\frac{J_{d}}{\left(1-\rho_{s}\right)}}{\frac{\rho_{d}}{\varepsilon \cdot A \cdot\left(1-\rho_{s}\right)}}
\end{aligned}
$$

which can be represented by the following network element:

$$
\begin{aligned}
& \stackrel{a}{E_{b}} \text { W } \\
& \frac{\rho_{d}}{\varepsilon \cdot A \cdot\left(1-\rho_{s}\right)}
\end{aligned}
$$

Fig. (11.) Network element with specular/diffuse reflectivities.

We now only consider diffuse exchanges between two surfaces 1 and 2:

$$
\begin{aligned}
& q_{1 \rightarrow 2, d}=J_{1, d} \cdot A_{1} \cdot F_{12} \cdot\left(1-\rho_{2, s}\right) \cdot \frac{\left(1-\rho_{1, s}\right)}{\left(1-\rho_{1, s}\right)} \\
& q_{2 \rightarrow 1, d}=J_{2, d} \cdot A_{2} \cdot F_{21} \cdot\left(1-\rho_{1, s}\right) \cdot \frac{\left(1-\rho_{2, s}\right)}{\left(1-\rho_{2, s}\right)} \\
& q_{12}^{\text {net }}=\frac{\frac{J_{1, d}}{\left(1-\rho_{1, s}\right)}-\frac{J_{2, d}}{\left(1-\rho_{2, s}\right)}}{A_{1} \cdot F_{12} \cdot\left(1-\rho_{1, s}\right) \cdot\left(1-\rho_{2, s}\right)}
\end{aligned}
$$

which can be represented by the following equivalent network element:

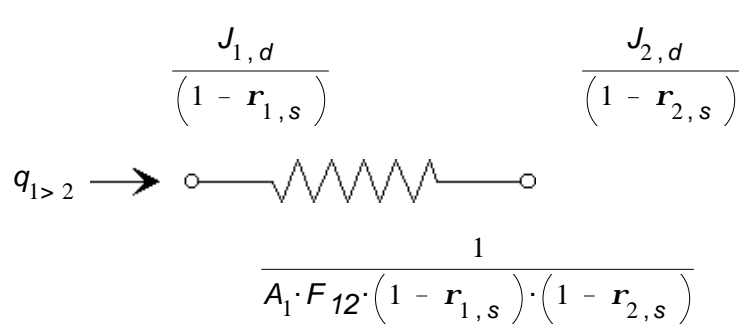

Fig. (12.) Equivalent network element for 2 surfaces with specular/diffuse reflectivities.

So the complete radiation heat transfer can be translated into an equivalent electrical network making use of the two precedent types of network element. The only additional adaptation is the replacement of the usual view factor by a specular view factor.

Indeed, in presence of partly specular surfaces, the notion of view factor must be modified. To define the notion of specular view factor in a system of partly diffuse/partly specular surfaces, we imagine in a first step that the diffuse part of the reflectivity of a surface acts as an absorber so that in a first step, there are only specular reflections, the diffused part of it is considered as absorbed and will be emitted later on, as a Lambertian emitter. In this situation, $F_{i j}^{s}$ represents the proportion of the energy diffusely emitted by surface $\mathrm{i}$ which after multiple specular reflections on the other surfaces, will finally be absorbed by surface $\mathrm{j}$.

$$
\begin{aligned}
F_{i j}^{s}= & F_{i j}+\sum_{l_{\text {specular }}} F_{i(j, l)} \cdot \rho_{l, s} \\
& +\sum_{l m_{\text {specular }}} F_{i[(j, l), m]} \cdot \rho_{l, s} \cdot \rho_{m, s}+\ldots
\end{aligned}
$$

where $[((j, \ldots) l), m]$ represents the image in the partly specular surface $\mathrm{m}$ of the image in the partly specular surface 1 , of the image in ... of the partly diffusely reflecting or absorbing surface $\mathrm{j}$.

The method which is used only consider net heat exchanges in a world exclusively composed of the diffuse or absorptive parts of the surfaces and their images; the specular part of the surfaces only makes images.

So, each surface i has an effective area of

$$
\left(1-\rho_{i, s}\right) \cdot A_{i}
$$

The view factors between diffuse surfaces (including their images) become:

$$
F_{i j} \cdot\left(1-\rho_{j, s}\right) \text { or } F_{i j}{ }^{s} \cdot\left(1-\rho_{j, s}\right)
$$

In this world of non specular surfaces and their images, we can redefine the absorption coefficients and the diffuse reflectivity's as

$$
\begin{aligned}
& \alpha_{i}^{\prime}=\frac{\alpha_{i}}{\left(1-\rho_{i, s}\right)} \\
& \rho_{\mathrm{i}, \mathrm{d}}{ }^{\prime}=1-\alpha_{i}^{\prime}=\frac{\rho_{i, d}}{\left(1-\rho_{i, s}\right)} \\
& F_{i j}^{\prime}=F_{i j} \cdot\left(1-\rho_{j, s}\right) \\
& F_{i j}^{s^{\prime}}=F_{i j}{ }^{s} \cdot\left(1-\rho_{j, s}\right) \\
& A_{i}^{\prime}=\left(1-\rho_{i, s}\right) \cdot A_{i}
\end{aligned}
$$

In this new world, we can apply the usual equations corresponding to a system of gray diffuse surfaces (conditions 1-4 are now verified).

$$
\begin{aligned}
& A_{i}^{\prime} \cdot F_{i j}^{\prime}=A_{j}^{\prime} \cdot F_{j i}^{\prime} \text { or } \\
& \left(1-\rho_{\mathrm{i}, \mathrm{s}}\right) \cdot A_{i} \cdot F_{i j} \cdot\left(1-\rho_{\mathrm{j}, \mathrm{s}}\right)=\left(1-\rho_{\mathrm{j}, \mathrm{s}}\right) \cdot A_{j} \cdot F_{j i} \cdot\left(1-\rho_{\mathrm{i}, \mathrm{s}}\right) \\
& A_{i}^{\prime} \cdot F_{i j}^{s^{\prime}}=A_{j}^{\prime} \cdot F_{j i}^{s^{\prime}} \text { or } \\
& \left(1-\rho_{\mathrm{i}, \mathrm{s}}\right) \cdot A_{i} \cdot F_{i j}^{s} \cdot\left(1-\rho_{\mathrm{j}, \mathrm{s}}\right)=\left(1-\rho_{\mathrm{j}, \mathrm{s}}\right) \cdot A_{j} \cdot F_{j i}^{s} \cdot\left(1-\rho_{\mathrm{i}, \mathrm{s}}\right)
\end{aligned}
$$

For those who could have doubts about the applicability of the symmetry relation in presence of curved specular reflectors, it could be recalled that the symmetry relation has its correspondent in optics: the Lagrangian invariant.

$$
\sum_{j_{d i f f i s e}} F_{i j}^{s^{\prime}}=1
$$




\section{ANNEX 3: GEBHART THEORY IN PRESENCE OF PARTLY SPECULAR / PARTLY DIFFUSE SURFACES}

The purpose of this paragraph is to generalize the Gebhart factors equation when all the surfaces satisfy the assumptions:

1. each surface is isothermal

2. each surface is a gray body

3. each surface is a diffuse emitter

4 '. each surface is a partly diffuse/partly specular reflector

5. the radiosity is uniformly distributed across each surface

The Gebhart factor $B_{i j}$ is the solution of the following set of equations:

$$
B_{i j}=F_{i j}^{s^{\prime}} \cdot \alpha_{j}^{\prime}+\sum_{\forall l_{d i f f i s e}} F_{i l}^{s^{\prime}} \cdot\left(1-\alpha_{l}^{\prime}\right) \cdot B_{l j}
$$

or in a matrix form :

$$
\underline{\underline{B}}=\underline{\underline{\beta}}+(\underline{\underline{F}}-\underline{\underline{\beta}}) \cdot \underline{\underline{B}}
$$

where $\beta_{i, j}=F_{i j}^{s} \cdot \alpha_{j}^{\prime}$

\section{Demonstration of the general properties of the}

\section{Gebhart factors in the case of diffuse / specular} reflections

Our aim is to redemonstrate the two general properties of the Gebhart factors:

1) $\varepsilon_{i}^{\prime} \cdot A_{i}^{\prime} \cdot B_{i, j}=\varepsilon_{j}^{\prime} \cdot A_{j}^{\prime} \cdot B_{j, i} ; \varepsilon_{i}^{\prime}=\frac{\varepsilon_{i}}{\left(1-\rho_{i, s}\right)}$

2) $\sum_{\forall j_{d i f f i s e}} B_{i, j}=1$ in the case of diffuse / specular

reflections, making use of the equation $\underline{\underline{B}}=\underline{\underline{\beta}}+(\underline{\underline{F}}-\underline{\underline{\beta}}) \cdot \underline{\underline{B}}$
Property 2:

$$
\sum_{\forall j_{\text {diffuse }}} B_{i, j}=1 ?
$$

or

$$
\sum_{j_{\text {diffuse }}} Y_{i j}=\varepsilon_{i} \cdot A_{i} \quad \text { or } \quad \underline{B} \cdot \underline{u}=\underline{u} ?
$$

where the vector $\underline{u}$ is defined by $u_{i}=1$ for $\forall \mathrm{i}$ The view factors satisfy the equation:

$$
\begin{aligned}
& \underline{\underline{F}} \underline{\underline{u}}=\underline{u} \text { (or } \sum_{j_{\text {diffuse }}} F_{i j}^{s}=1 \text { for } \forall \mathrm{i} \text { ) } \\
& \underline{\underline{B}}=\underline{\underline{[\mathrm{I}-\mathrm{F}+\beta]^{-1}}} \cdot \underline{\underline{\beta}} \\
& \underline{\underline{\beta}}=\underline{\underline{[I-F+\beta}]}-\underline{\underline{[I-F]}} \\
& \underline{\underline{B}}=\underline{\underline{I-[I-F+\beta]^{-1}}} \cdot \underline{\underline{[I-F]}} \\
& \underline{\underline{B}} \underline{u}=\underline{\underline{u}}-\underline{\underline{[1-F+\beta]^{-1}}} \cdot[\underbrace{\underline{u}-\underline{\underline{F}} \underline{u}}_{=0}]
\end{aligned}
$$$$
\text { so } \quad \underline{B} \underline{\underline{u}}=\underline{u}
$$

\section{Property 1:}

$$
\begin{aligned}
\varepsilon_{i}^{\prime} \cdot A_{i}^{\prime} \cdot B_{i, j}= & \varepsilon_{i}^{\prime} \cdot A_{i}^{\prime} \cdot F_{i, j}^{s^{\prime}} \cdot \varepsilon_{j}^{\prime} \\
& +\sum_{l_{d i f f i s e}} \varepsilon_{i}^{\prime} \cdot A_{i}^{\prime} \cdot F_{i l}^{s^{\prime}} \cdot \varepsilon_{l}^{\prime} \cdot\left(\frac{1-\varepsilon_{l}^{\prime}}{\varepsilon_{l}^{\prime}}\right) \frac{\varepsilon_{l}^{\prime} \cdot A_{l}^{\prime}}{\varepsilon_{l}^{\prime} \cdot A_{l}^{\prime}} B_{l, j}
\end{aligned}
$$

or $\quad \underline{\underline{Y}}=\underline{\underline{U}}+\underline{\underline{U}} \cdot \underline{\underline{G}} \cdot \underline{\underline{Y}}$

where $U_{i j}=\varepsilon_{i}^{\prime} \cdot A_{i}^{\prime} \cdot F_{i j}^{s^{\prime}} \cdot \varepsilon_{j}^{\prime}=U_{j i}, G=\operatorname{diag}\left(\frac{1-\varepsilon^{\prime}}{\varepsilon^{\prime 2} \cdot A^{\prime}}\right)$.

$\underline{\underline{U}}=\underline{\underline{U}}^{T} ; \underline{\underline{G}}=\underline{\underline{G}}^{T} \Rightarrow \underline{\underline{Y}}=\underline{\underline{Y}}^{T}$

since $\underline{\underline{Y}}=\underline{\underline{U}}+\underline{\underline{U}} \underline{\underline{G}} \underline{\underline{U}}+\underline{\underline{U}} \underline{\underline{G}} \underline{\underline{U}} \underline{\underline{G}} \underline{\underline{U}}+\cdots$ where each term is symmetrical so Property 1 is demonstrated 